trueream

* Gated RapidArc

* Five Photon Beam

* High Dose Rate : 2400MU/min

* Supervisor System

* 4D CBCT and Cine Mode

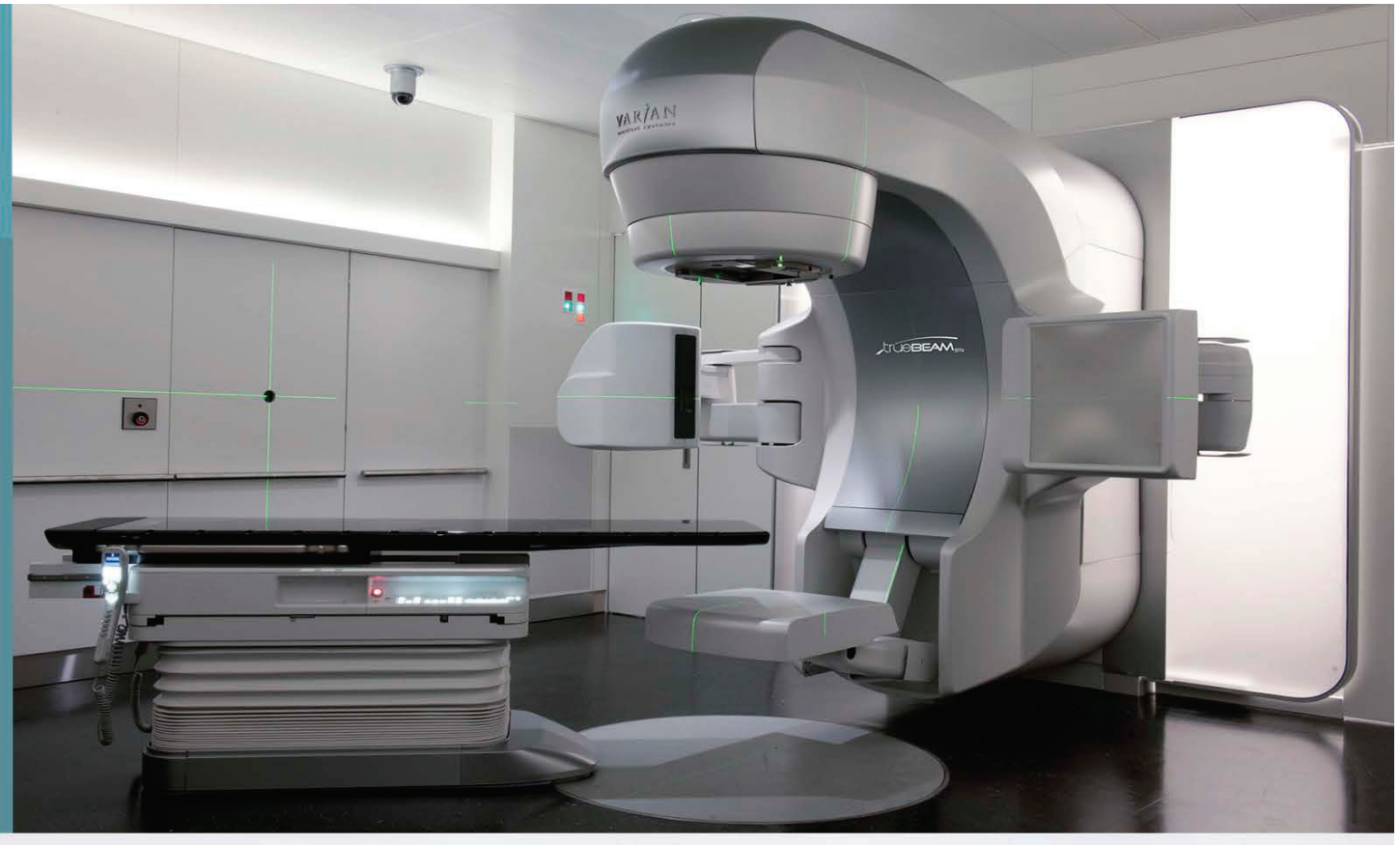

\section{-Vit:oll $\widehat{E A M}$}

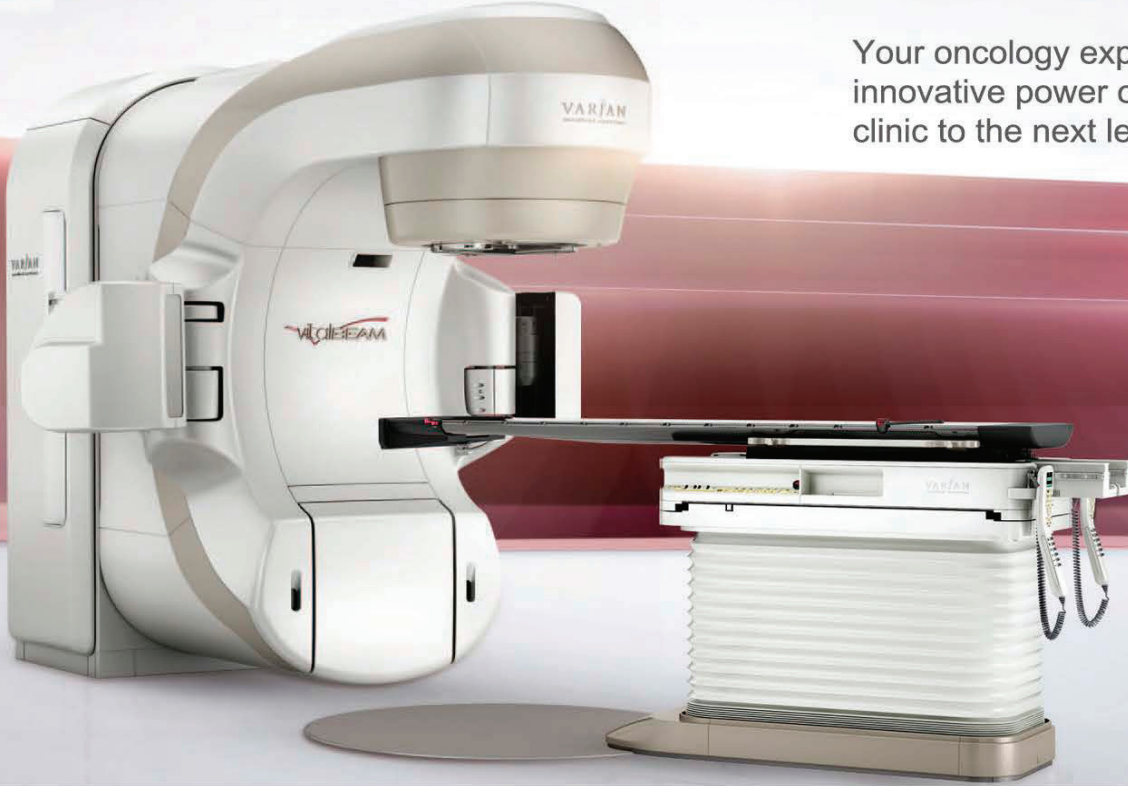

\section{VITAL TOGETHER}

Your oncology expertise combined with the innovative power of VitalBeam can help take your
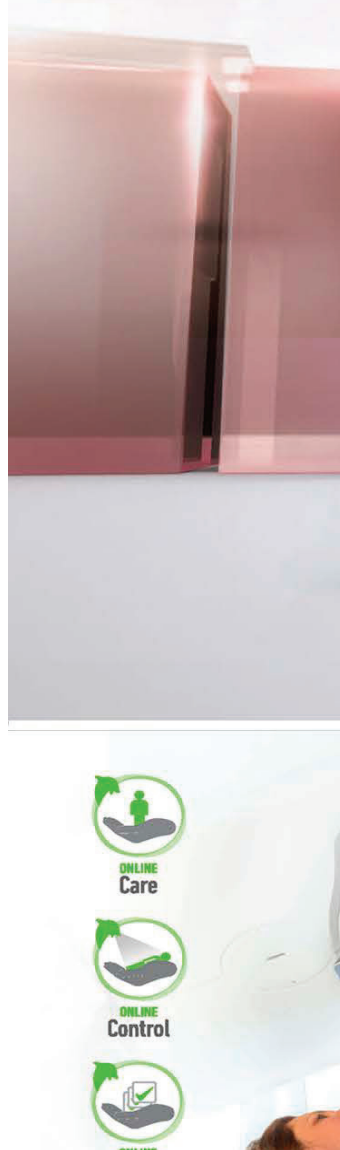
Confidence
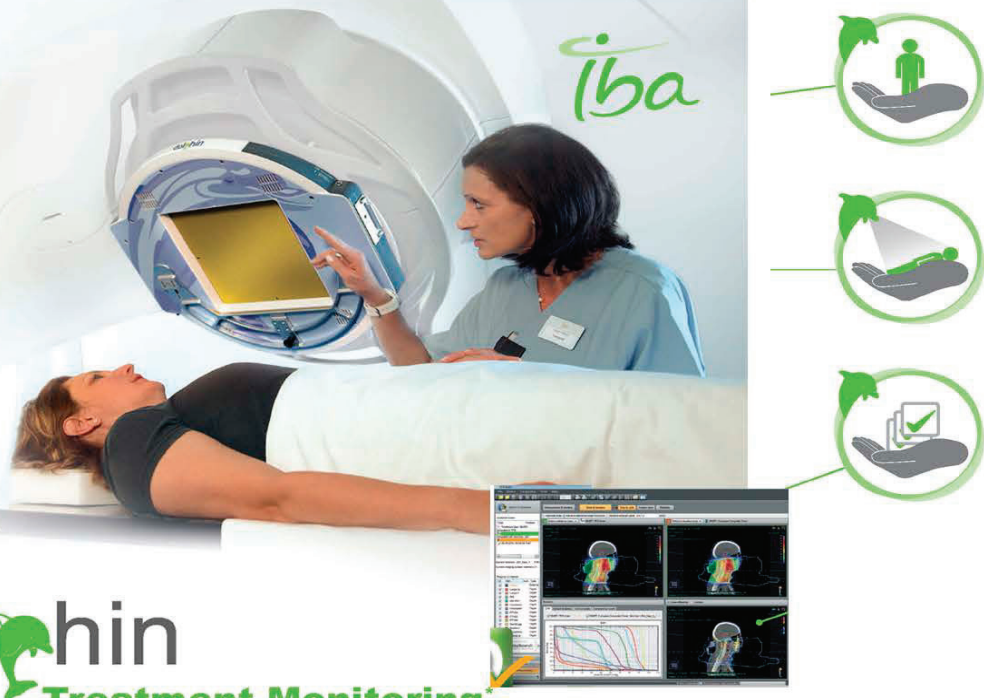

Offer your patients more care and comfort to give them hope, belief and "the will to fight."

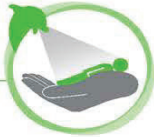

Be certain you have control over your patients' safety against accidents !

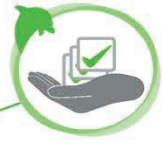

Have the confidence and peace of mind knowing that your treatment is accurate until the last fraction!

\section{dolphin}

Online Treatment Monitoring
$2 \underset{\text { Larparation }}{2}$

서울시 종로구 인사동 194-27

TEL : 02) 2003-8441

FAX : 02) $737-4677$ 


\title{
Acceptance Test and Clinical Commissioning of CT Simulator
}

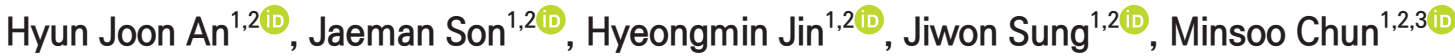 \\ ${ }^{1}$ Department of Radiation Oncology, ${ }^{2}$ Biomedical Research Institute, Seoul National University Hospital, ${ }^{3}$ Institute of Radiation Medicine, \\ Seoul National University Medical Research Center, Seoul, Korea
}

Received 13 December 2019 Revised 17 December 2019 Accepted 18 December 2019

Corresponding author

Minsoo Chun

(ms1236@snu.ac.kr)

Tel: 82-2-2072-4151

Fax: 82-2-765-3317
This study examined the clinical use of two newly installed computed tomography (CT) simulators in the Department of Radiation Oncology. The accreditation procedure was performed by the Korean Institute for Accreditation of Medical Imaging. An Xi R/F dosimeter was used to measure the CT dose index for each plug of the CT dose index phantom. Image qualities such as the Hounsfield unit $(\mathrm{HU})$ value of water, noise level, homogeneity, existence of artifacts, spatial resolution, contrast, and slice thickness were evaluated by scanning a CT performance phantom. All test items were evaluated as to whether they were within the required tolerance level. CT calibration curves-the relationship between $\mathrm{CT}$ number and relative electron density-were obtained for dose calculations in the treatment planning system. The positional accuracy of the lasers was also evaluated. The volume CT dose indices for the head phantom were $22.26 \mathrm{mGy}$ and $23.70 \mathrm{mGy}$, and those for body phantom were $12.30 \mathrm{mGy}$ and $12.99 \mathrm{mGy}$ for the first and second CT simulators, respectively. $\mathrm{HU}$ accuracy, noise, and homogeneity for the first $\mathrm{CT}$ simulator were $-0.2 \mathrm{HU}, 4.9 \mathrm{HU}$, and $0.69 \mathrm{HU}$, respectively, while those for second CT simulator were $1.9 \mathrm{HU}$, $4.9 \mathrm{HU}$, and $0.70 \mathrm{HU}$, respectively. Five air-filled holes with a diameter of $1.00 \mathrm{~mm}$ were used for assessment of spatial resolution and a low contrast object with a diameter of $6.4 \mathrm{~mm}$ was clearly discernible by both CT scanners. Both CT simulators exhibited comparable performance and are acceptable for clinical use.

Keywords: CT simulator, Acceptance test, CT dose index, Image quality

\section{Introduction}

Since the late 1970s, computed tomography (CT) has been widely used for radiotherapy planning with the advantages of improved patient positioning, target delineation, treatment beam arrangement, and dose calculation. $^{1-3)}$ Unlike a diagnostic CT scanner, a wide bore $(>80$ $\mathrm{cm}$ ) scanner is generally used for CT simulations. This is largely to accommodate extremely large patients, breast cancer patients for whom the ipsilateral arm is subtended to approximately $90^{\circ}$, and patients with specialized im- mobilization devices. ${ }^{4,5)}$ Furthermore, a flat-bed couch and moving laser system are required for treatment simulation. ${ }^{5)}$ Notably, the characteristics and performance of a radiation oncology-dedicated CT scanner should be validated in advance of patients' CT simulations to ensure accuracy. $^{5,6)}$

According to the American College of Radiology (ACR) CT accreditation program, a submission of clinical and phantom images, dose measurements and scanning protocols is mandatory for any institution using CT devices in the United States. ${ }^{7)}$ Image qualities such as CT number 
Table 1. Verification of scan parameters

\begin{tabular}{|c|c|c|c|c|c|c|c|}
\hline \multicolumn{4}{|c|}{ Scan parameter setting } & \multicolumn{2}{|c|}{$\mathrm{kV}$} & \multicolumn{2}{|c|}{$\mathrm{mAs}^{*}$} \\
\hline $\mathrm{kV}$ & $\mathrm{mA}$ & Seconds & mAs & CT SIM1 & CT SIM2 & CT SIM1 & CT SIM2 \\
\hline 80 & 300 & 0.5 & 150 & 80.416 & 80.537 & 151.592 & 152.616 \\
\hline 80 & 200 & 1 & 200 & 80.616 & 80.726 & 201.348 & 201.648 \\
\hline 80 & 50 & 2 & 100 & 80.050 & 81.115 & 100.434 & 101.413 \\
\hline 120 & 300 & 0.5 & 150 & 122.091 & 122.441 & 150.360 & 151.618 \\
\hline 120 & 200 & 1 & 200 & 122.458 & 122.266 & 200.373 & 201.108 \\
\hline 120 & 50 & 2 & 100 & 122.258 & 122.338 & 100.233 & 101.228 \\
\hline 140 & 300 & 0.5 & 150 & 141.295 & 142.006 & 150.170 & 151.430 \\
\hline 140 & 200 & 1 & 200 & 141.928 & 142.778 & 200.480 & 200.901 \\
\hline 140 & 50 & 2 & 100 & 143.029 & 143.823 & 100.300 & 101.278 \\
\hline
\end{tabular}

CT, computed tomography; SIM1, the first CT simulator; SIM2, the second CT simulator.

*Product of tube current and time.

accuracy, low-contrast resolution, image uniformity, and volume CT dose index $\left(\mathrm{CTDI}_{\mathrm{vol}}\right)$ are assessed in the accreditation process. The acceptance criteria for $\mathrm{CTDI}_{\mathrm{vol}}$ are $80 \mathrm{mGy}$ and $30 \mathrm{mGy}$ for adult head and abdominal scans, respectively. ${ }^{7)}$ In Europe, a reference level of $60 \mathrm{mGy}$ for weighted CTDI was proposed for a routine head protocol. ${ }^{8)}$ A similar accreditation program is performed in South Korea by the Korean Institute for Accreditation of Medical Imaging (KIAMI). For this program, spatial resolution, low contrast resolution, linearity, image noise, and the presence of artifacts are evaluated along with CTDI for each plug position. ${ }^{9)}$ The CTDI acceptance criterion for each plug position is much stricter in South Korea than those stated by international guidelines. Although $\mathrm{CTDI}_{\mathrm{vol}}$ does not imply the actual patient dose, ${ }^{10,11)}$ CTDI $_{\mathrm{vol}}$ can be used for the verification and monitoring of imaging doses. ${ }^{7-9)}$ Although several studies insist that noise is the primary variable affecting image quality and diagnostic performance, ${ }^{12,13)}$ other quality indices should also be examined for CT scanner assessment. ${ }^{5,14)}$

Recently, two CT simulators were newly installed in our institute. The aim of this study was thus to evaluate the image quality and radiation dose of these CT simulators, and to ensure their clinical acceptance for use in radiotherapy planning.

\section{Materials and Methods}

Two Brilliance CT Big Bore (Philips Medical System, Cleveland, $\mathrm{OH}, \mathrm{USA}$ ) systems were newly installed in our institute for CT simulation for radiotherapy. The accreditation procedures were performed by the KIAMI during the acceptance testing stage of the CT simulators.

\section{Scan parameters and computed tomography dose index}

The scan parameters were evaluated to confirm whether the $\mathrm{kV}$ and $\mathrm{mAs}$ set by users are appropriately and accurately delivered by the $\mathrm{CT}$ simulators. An Xi R/F detector (Unfors RaySafe AB, Billdal, Sweden) capable of measuring tube potential, dose, dose rate, pulse, pulse rate, dose/ frame, time, half-value layer, and total filtration simultaneously was used for this purpose. Scan parameters are listed in Table 1, and the tolerance limits for the measurements were $\pm 7 \%$ and $\pm 10 \%$ for $\mathrm{kVp}$ and mAs, respectively. CTDI was measured with dedicated a head and body CTDI phantom (Fluke Corporation, Everett, WA, USA). An Xi CT detector (Unfors RaySafe $\mathrm{AB}$ ) and a black piranha Quality Assurance (QA) meter (RTI Electronics AB, Moelndal, Sweden) were used. CTDIs for each plug position were evaluated as to whether the measurement was within the acceptable range (Table 2 ).

\section{Image quality}

A modular 76-410 AAPM CT Performance Phantom (Fluke Corporation; Fig. 1a) was scanned to evaluate image quality. The phantom contained a contrast test object, a CT number linearity insert, a resolution insert, and a 
slice thickness insert. The phantom was scanned with 120 $\mathrm{kVp}, 250 \mathrm{mAs}$, and a slice thickness of $10 \mathrm{~mm}$, and was reconstructed with a standard reconstruction algorithm. The Hounsfield unit (HU) of water, noise level, homogeneity, presence of artifacts, spatial resolution, contrast, and slice thickness were evaluated in the reconstructed images. The mean and standard deviation of $4 \times 4 \mathrm{~cm}^{2}$ regions of interest (ROI) drawn in water were defined as the HU of water and noise level, respectively. The HU of water should be between $-7 \mathrm{HU}$ and $7 \mathrm{HU}$, and the noise level should be less than 7 HU. Homogeneity was calculated

Table 2. CTDI measured with an Xi CT detector

\begin{tabular}{llrrr}
\hline \multirow{2}{*}{ Phantom } & \multirow{2}{*}{ Location } & \multirow{2}{*}{$\begin{array}{c}\text { Acceptable } \\
\text { range }\end{array}$} & \multicolumn{2}{c}{ CTDI (mGy) } \\
\cline { 3 - 5 } & & \multicolumn{1}{c}{ CT SIM1 } & CT SIM2 \\
\hline \multirow{2}{*}{ Head } & Center & $24.8 \mathrm{mGy} \pm 35 \%$ & 21.059 & 21.867 \\
& $0^{\circ}(12$ o'clock) & $28.7 \mathrm{mGy} \pm 35 \%$ & 24.713 & 25.886 \\
& $90^{\circ}(3$ o'clock) & $27.3 \mathrm{mGy} \pm 35 \%$ & 23.079 & 24.597 \\
& $180^{\circ}(6$ o'clock) & $25.8 \mathrm{mGy} \pm 35 \%$ & 21.146 & 22.633 \\
\multirow{3}{*}{ Body } & $270^{\circ}(9$ o'clock) & $27.4 \mathrm{mGy} \pm 35 \%$ & 22.520 & 25.375 \\
& Center & $8.7 \mathrm{mGy} \pm 35 \%$ & 7.362 & 7.383 \\
& $0^{\circ}(12$ o'clock $)$ & $18.4 \mathrm{mGy} \pm 35 \%$ & 16.336 & 16.745 \\
& $90^{\circ}(3$ o'clock $)$ & $18.5 \mathrm{mGy} \pm 35 \%$ & 15.144 & 15.957 \\
& $180^{\circ}$ (6 o'clock) & $18.1 \mathrm{mGy} \pm 35 \%$ & 12.326 & 13.647 \\
& $270^{\circ}$ (9 o'clock) & $16.3 \mathrm{mGy} \pm 35 \%$ & 15.274 & 16.802 \\
\hline
\end{tabular}

Both scans were taken with $120 \mathrm{kVp}, 250 \mathrm{mAs}$, and $2.4 \mathrm{~cm}$ of slice thickness. Values are presented as mean \pm standard deviation. CTDI, computed tomography dose index; CT, computed tomography; SIM1, the first CT simulator; SIM2, the second CT simulator. as the standard deviation of HU for 4 ROIs and should be less than 5 HU. The existence of artifacts was subjectively evaluated by determining if reviewers could identify any visible artifacts. In the image of the spatial resolution insert (Fig. 1c) where eight sets of five air-filled holes are imaged (with diameters of 1.75, 1.5, 1.25, 1.00, 0.75, 0.61, 0.5, and $0.4 \mathrm{~mm}$ ), reviewers should be able to discern all five holes of each set at least $1.00 \mathrm{~mm}$ diameter in order to pass the spatial resolution test. Six pairs of the low contrast inserts (Fig. 1d) with diameters of 25.4, 19.1, 12.7, 9.5, 6.4, and 3.2 $\mathrm{mm}$ were filled with dextrose or sodium chloride solutions of various densities with $2 \%-3 \%$ differentials in density between cavities. Reviewers must be able to identify the two $6.4 \mathrm{~mm}$ spheres in the image in order to pass the low contrast resolution test. The slice thickness as determined by measuring the distance of the aluminum strips should be accurate within $\pm 1 \mathrm{~mm}$ to pass the slice thickness test (Fig. 1e). Complete assessments were performed independently by two KIAMI-trained reviewers.

\section{Hounsfield unit curves and laser position}

An electron density phantom (Model 62M; CIRS Inc., Norfolk, VA, USA) was used to acquire the HU value corresponding to each tissue-equivalent insert. The phantom was scanned with a body scan protocol of $120 \mathrm{kVp}, 213$ $\mathrm{mAs}$, and $2 \mathrm{~mm}$ slice thickness. The acquired HU to electron density curve and the acquired HU to physical density
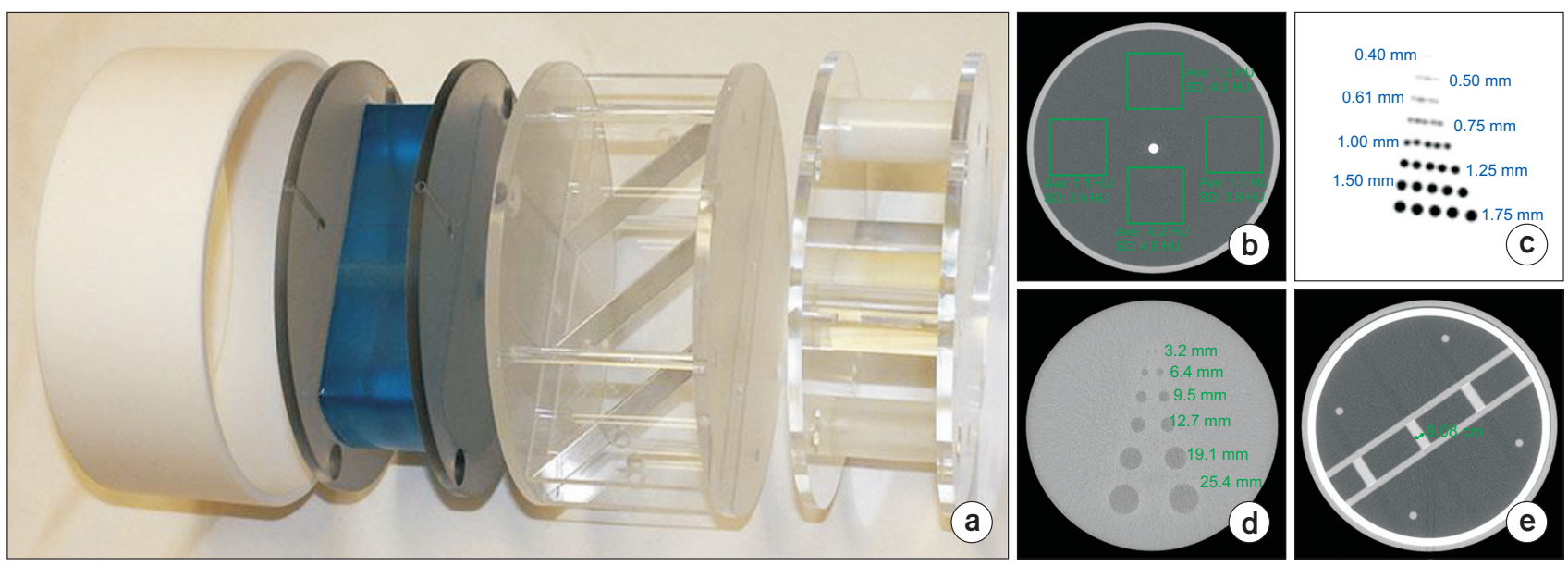

Fig. 1. (a) A modular 76-410 AAPM computed tomography (CT) Performance Phantom (Fluke Corporation, Everett, WA, USA); CT slices of (b) water Hounsfield unit (HU), noise level, homogeneity evaluation, (c) spatial resolution, (d) low contrast resolution insert, and (e) slice thickness. Ave, average; SD, standard deviation. 
Table 3. Physical characteristics and HU acquired during the clinical commissioning stage

\begin{tabular}{|c|c|c|c|c|c|}
\hline Plug bar description & $\begin{array}{l}\text { Physical density } \\
(\mathrm{g} / \mathrm{mL})\end{array}$ & $\begin{array}{c}\text { Electron density } \\
\left(\times 10^{23} \text { electrons } / \mathrm{mL}\right)\end{array}$ & $\begin{array}{l}\text { Relative electron density } \\
\text { (relative to } \mathrm{H}_{2} \mathrm{O} \text { ) }\end{array}$ & HU for CT SIM1 & HU for CT SIM2 \\
\hline Lung inhale & 0.20 & 0.634 & 0.190 & -795.3 & -792.1 \\
\hline Lung exhale & 0.50 & 1.632 & 0.489 & -457.2 & -458.8 \\
\hline Adipose & 0.96 & 3.171 & 0.949 & -65.2 & -67.4 \\
\hline Breast ( $50 \%$ gland $/ 50 \%$ adipose) & 0.99 & 3.261 & 0.976 & -33.3 & -31.6 \\
\hline Muscle & 1.06 & 3.483 & 1.043 & 50.6 & 50.2 \\
\hline Liver & 1.07 & 3.516 & 1.052 & 56.3 & 59.2 \\
\hline Trabecular bone (200 mg/mL) & 1.16 & 3.730 & 1.117 & 213.1 & 216.4 \\
\hline Solid dense bone ( $800 \mathrm{mg} / \mathrm{mL})$ & 1.53 & 4.862 & 1.456 & 867.8 & 868.2 \\
\hline
\end{tabular}

HU, Hounsfield unit; CT, computed tomography; SIM1, the first CT simulator; SIM2, the second CT simulator.

curve were imported to the treatment planning system for dose calculation.

A DORADOnova 3 laser system (LAP GmbH Laser Applications, Lüneburg, Germany) along with a CARINAnav smart control system (LAP GmbH Laser Applications) for patient alignment were installed. A Wilke phantom was aligned using the DORADOnova 3 laser system, and scanned with $120 \mathrm{kV}, 200 \mathrm{mAs}$, and a slice thickness of 1.25 $\mathrm{mm}$. The accuracy of the laser system was analyzed using the tumorLOC program (Philips Medical System).

\section{Results}

\section{Verification of scan parameters and computed tomography dose index}

The scan parameters set by users were correctly measured, showing average discrepancies of $-1.28 \%$ and $-1.67 \%$ in $\mathrm{kV}$, and $-0.39 \%$ and $-1.06 \%$ in $\mathrm{mAs}$, for the first CT simulator (CT SIM1) and the second CT simulator (CT SIM2), respectively. As shown in Table 1, the results of the scan parameter verification were tolerable for both CT simulators as they were within the tolerance levels of $\pm 7 \%$ and $\pm 10 \%$ for $\mathrm{kVp}$ and $\mathrm{mAs}$, respectively. The CTDI measurements are provided in Table 2 . The head and body CTDIs for each chamber position were all within the tolerance limit for both CT simulators.

\section{Image quality}

As shown in Fig. 1b, the HU of water, the noise level, and the homogeneity were $-0.2 \mathrm{HU}, 4.9 \mathrm{HU}$, and $0.69 \mathrm{HU}$, respectively, for CT SIM1. These parameters for CT SIM2 were $1.9 \mathrm{HU}, 4.9 \mathrm{HU}$, and 0.70 HU, respectively. All reviewers agreed that there were no visible artifacts as shown in Fig. 1b. Five air-filled holes with a diameter of $1.00 \mathrm{~mm}$ and a low contrast object with a diameter of $6.4 \mathrm{~mm}$ were clearly discernible for both CT simulators as shown in Fig. 1c, d, respectively. Thus, the CT simulators passed the spatial resolution assessment. The slice thicknesses for the aluminum strip were $8.9 \mathrm{~mm}$ and $9.1 \mathrm{~mm}$, for CT SIM1 and CT SIM2, respectively. All testing parameters were within the required tolerance level (Fig. 1e).

\section{Hounsfield unit curves and verification of laser position}

The phantom manufacturer provided the physical density as well as the electron density for the designated materials. The average $\mathrm{HU}$ values for each ROI according to the material were comparable for both scanners. A relatively large HU difference for the lung inhale insert (3.2 HU) and at the trabecular bone insert (3.3 HU) was observed, and the complete results are summarized in Table 3 . The average of $\mathrm{HU}$ values corresponding to each relative electron density (RED) were imported into the treatment planning system. The laser position was verified in three directions as shown in Fig. 2. The difference between the laser position and the groove center shown in the images was less than $0.1 \mathrm{~mm}$ for both scanners. 

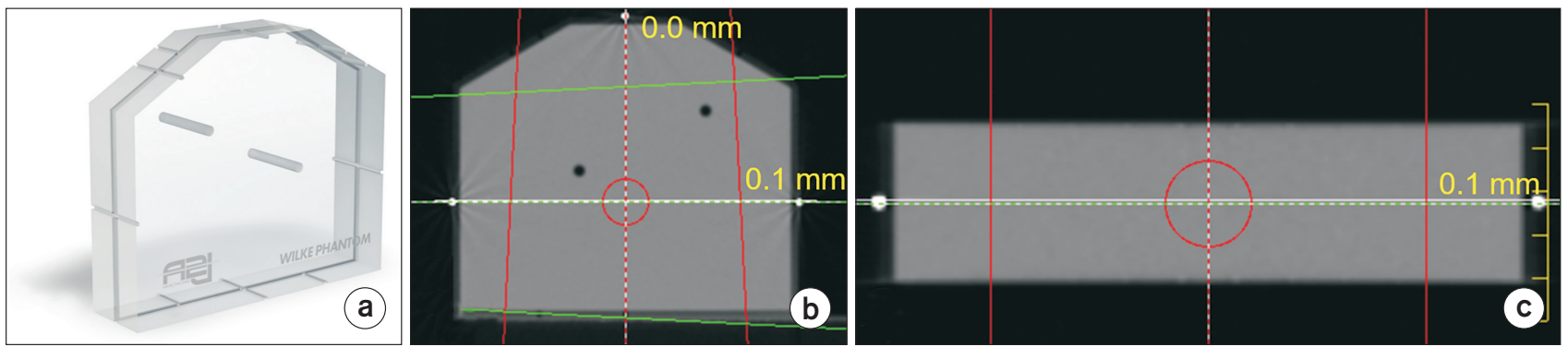

Fig. 2. (a) Wilke phantom, (b) an axial slice, and (c) a coronal slice. The deviations between laser position and groove position in the images are marked.

\section{Discussion}

The accreditation procedure for the newly installed CT simulators was performed by reviewers in KIAMI. All test items were identified as acceptable for both scanners. CTDI is often not recommended as a surrogate for patient doses as it does not consider an actual body size or composition. ${ }^{10,11,15-17)}$ Despite this, CTDI measurement is crucial because it is applied when estimating a more precise dose estimate such as size-specific dose estimates. ${ }^{16,17)}$ As mentioned above, the tolerance levels for $\mathrm{CTDI}_{\mathrm{vol}}$ suggested by the ACR accreditation program are $80 \mathrm{mGy}$ and $30 \mathrm{mGy}$ for a head and a body phantom, respectively. By applying the $\mathrm{CTDI}_{\mathrm{vol}}=1 / 3 \times \mathrm{CTDI}_{\text {center }}+2 / 3 \times \mathrm{CTDI}_{\text {periphery }}$ formula, the CT$\mathrm{DIs}_{\mathrm{vol}}$ in KIAMI were $35.73 \mathrm{mGy}$ and $19.96 \mathrm{mGy}$ for a head and a body phantom, respectively. In our head phantom measurements, the measured CTDIs $\mathrm{vol}_{\mathrm{vl}}$ were $22.26 \mathrm{mGy}$ and 23.70 mGy for CT SIM1 and CT SIM2, respectively. Furthermore, for body phantom measurements, the CTDIs were $12.30 \mathrm{mGy}$ and $12.99 \mathrm{mGy}$ for CT SIM1 and CT SIM2, respectively. All measurements were within strict tolerance levels.

HU uniformity, noise level, and homogeneity were also within required tolerance levels. The acceptance criteria for noise level provided by KIAMI changed from $8 \mathrm{HU}$ to 7 $\mathrm{HU}$ in 2007. Noise level is known to dominate overall image quality, thus the detectability of a lesion is mainly affected by noise level. ${ }^{18,19)}$ According to existing literature regarding image quality in multiple CT scanners, higher noise levels resulted in lower contrast-to-noise ratio., ${ }^{9,14)}$ Therefore, the application of stricter criteria for noise level is reasonable.

A CT calibration curve-that is, the relationship between HU values and their corresponding RED-is necessary in dose calculations as megavoltage photon beams interact primarily with Compton scattering. ${ }^{3,20)}$ Although CT numbers are defined as being proportional to the linear attenuation coefficient, individual scanners exhibit inaccuracies. ${ }^{6,21)}$ Therefore, the HU corresponding to each material should be measured for a given scanner, which is generally established during the commissioning stage. ${ }^{6)}$ Notably, the consistency of HU for a given tissue should be monitored because changes in $\mathrm{HU}$ will result in changes in dose distribution. ${ }^{6,22)}$ Typically, HU value changes of $\pm 20 \mathrm{HU}$ for soft tissue and $\pm 50 \mathrm{HU}$ for lung and bone are known to lead to $1 \%$ changes in dose distribution. ${ }^{22)}$ The two newly installed CT simulators showed consistent HU values showing a maximum difference of $3.3 \mathrm{HU}$. Since this difference is negligible when considering the tolerance for HU consistency, a single CT calibration curve by averaging two HUs was applied in the treatment planning system.

In short, installation, acceptance testing, and commissioning of the new CT simulators were performed. Although the acceptance testing and commissioning of the CT simulators were tolerable, periodic quality assurance should be performed by medical physicists. ${ }^{5,6)}$ The overall system should be appropriately monitored to rigorously maintain the CT simulators in the radiation oncology department.

\section{Conclusions}

The clinical use of the newly installed CT simulators was validated by performing image quality evaluation and dose measurements. Both CT simulators showed comparable performance and are acceptable for clinical use. 


\section{Acknowledgements}

This work was supported by the National Research Foundation of Korea (NRF) grant funded by the Korean government (NRF-2019R1F1A1041944).

\section{Conflicts of Interest}

The authors have no conflicts of interest to declare.

\section{Availability of Data and Materials}

All relevant data are within the paper and its Supporting Information files.

\section{References}

1. Geise RA, McCullough EC. The use of CT scanners in megavoltage photon-beam therapy planning. Radiology. 1977;124:133-141.

2. Dobbs HJ, Parker RP, Hodson NJ, Hobday P, Husband JE. The use of CT in radiotherapy treatment planning. Radiother Oncol. 1983;1:133-141.

3. Parker RP, Hobday PA, Cassell KJ. The direct use of CT numbers in radiotherapy dosage calculations for inhomogeneous media. Phys Med Biol. 1979;24:802-809.

4. Garcia-Ramirez JL, Mutic S, Dempsey JF, Low DA, Purdy JA. Performance evaluation of an 85-cm-bore X-ray computed tomography scanner designed for radiation oncology and comparison with current diagnostic CT scanners. Int J Radiat Oncol Biol Phys. 2002;52:1123-1131.

5. Mutic S, Palta JR, Butker EK, Das IJ, Huq MS, Loo LN, et al. Quality assurance for computed-tomography simulators and the computed-tomography-simulation process: report of the AAPM Radiation Therapy Committee Task Group No. 66. Med Phys. 2003;30:2762-2792.

6. Bissonnette JP, Balter PA, Dong L, Langen KM, Lovelock DM, Miften M, et al. Quality assurance for image-guided radiation therapy utilizing CT-based technologies: a report of the AAPM TG-179. Med Phys. 2012;39:1946-1963.

7. American College of Radiology. CT accreditation program requirements. American College of Radiology. 2019.

8. European Commission. European guidelines on quality criteria for computed tomography. European Commission. 1999.

9. Park HJ, Jung SE, Lee YJ, Cho WI, Do KH, Kim SH, et al. The relationship between subjective and objective parameters in CT phantom image evaluation. Korean J Radiol. 2009;10:490-495.

10. Deak PD, Smal Y, Kalender WA. Multisection CT protocols: sex- and age-specific conversion factors used to determine effective dose from dose-length product. Radiology. 2010; 257:158-166.

11. McCollough CH, Leng S, Yu L, Cody DD, Boone JM, McNitt-Gray MF. CT dose index and patient dose: they are not the same thing. Radiology. 2011;259:311-316.

12. Chun M, Choi YH, Kim JH. Automated measurement of CT noise in patient images with a novel structure coherence feature. Phys Med Biol. 2015;60:9107-9122.

13. SSchuhbaeck A, Schaefer M, Marwan M, Gauss S, Muschiol G, Lell M, et al. Patient-specific predictors of image noise in coronary CT angiography. J Cardiovasc Comput Tomogr. 2013;7:39-45.

14. Tomic N, Papaconstadopoulos P, Aldelaijan S, Rajala J, Seuntjens J, Devic S. Image quality for radiotherapy CT simulators with different scanner bore size. Phys Med. 2018;45:65-71.

15. Seibert JA, Boone JM, Wootton-Gorges SL, Lamba R. Dose is not always what it seems: where very misleading values can result from volume CT dose index and dose length product. J Am Coll Radiol. 2014;11:233-237.

16. Boone JM, Strauss KJ, Cody DD, McCollough CH, McNittGray MF, Toth TL et al. Size-specific dose estimates (SSDE) in pediatric and adult body CT examinations: the report of AAPM task group 204. Med Phys. 2011.

17. Mccollough C, Bakalyar DM, Bostani M, Brady S, Boedeker K, Boone JM et al. Use of water equivalent diameter for calculating patient size and size-specific dose estimates (SSDE) in CT: the report of AAPM task group 220. Med Phys. 2014.

18. Eck BL, Fahmi R, Brown KM, Zabic S, Raihani N, Miao J, et al. Computational and human observer image quality evaluation of low dose, knowledge-based CT iterative reconstruction. Med Phys. 2015;42:6098-6111.

19. Verdun FR, Racine D, Ott JG, Tapiovaara MJ, Toroi P, Bochud FO, et al. Image quality in CT: from physical mea- 
surements to model observers. Phys Med. 2015;31:823-843.

20. Battista JJ, Bronskill MJ. Compton scatter imaging of transverse sections: an overall appraisal and evaluation for radiotherapy planning. Phys Med Biol. 1981;26:81-99.

21. IAEA TRS Report 430. Commissioning and quality assurance of computerized planning systems for radiation treatment of cancer. Austria: International Atomic Energy Agency. 2004; 430.

22. Davis AT, Palmer AL, Nisbet A. Can CT scan protocols used for radiotherapy treatment planning be adjusted to optimize image quality and patient dose? A systematic review. Br J Radiol. 2017;90:20160406. 